The International Conference on Metallurgical Coatings and Thin Films

LBNL-51568

(ICMCTF 2003)

$\underline{\text { Paper B2-7 }}$

Note: This manuscript was presented but not submitted for publication. It remained a "formal report"

\title{
Effect of underlayer on coalescence of silver islands grown \\ by filtered cathodic arc deposition
}

\author{
Eungsun Byon ${ }^{\text {a) }}$ and André Anders ${ }^{\text {b) }}$ \\ ${ }^{a)}$ Korea Institute of Machinery and Materials, 66 Sangnam-Dong, \\ Changwon, 641-010, Korea \\ ${ }^{b)}$ Lawrence Berkeley National Laboratory, University of California, \\ 1 Cyclotron Road, Berkeley, California 94720-8223
}

March 1, 2003.

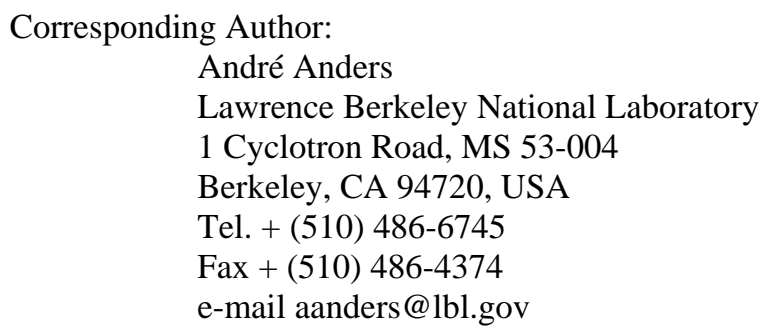

This work was supported by the Assistant Secretary for Energy Efficiency and Renewable Energy, Office of Building Technology, U.S. Department of Energy under Contract No. DE-AC03-76SF00098. Eungsun Byon was supported by the post-doctoral fellowship program of the Korean Science and Engineering Foundation (KOSEF). 


\title{
Effect of underlayer on coalescence of silver islands grown by filtered cathodic arc deposition
}

\author{
Eungsun Byon ${ }^{\text {a) }}$ and André Anders ${ }^{\text {b) }}$ \\ ${ }^{a)}$ Korea Institute of Machinery and Materials, 66 Sangnam-Dong, Changwon, 641-010, Korea

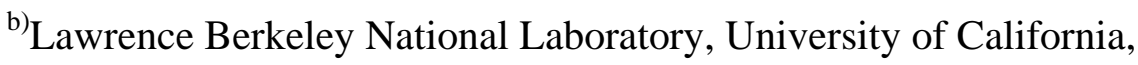 \\ 1 Cyclotron Road, Berkeley, California 94720-8223
}

\begin{abstract}
For low-emissivity application on window glass, coalescence of thin film silver islands is crucial for high transmittance in the visible and high reflectance in the infrared. It is well known that the energy of ions arriving at the substrate (kinetics) as wells as the type of underlayer (thermodynamics) affect the nucleation and growth mode. Little is known about coalescence of silver islands synthesized by energetic condensation, e.g. by filtered cathodic vacuum arc deposition. In this work, the effect of the underlayer on nucleation and growth of silver films deposited by filtered cathodic vacuum arc was investigated by transmission electron microscopy (TEM) and atomic force microscopy (AFM). The results are compared with data obtained on magnetron sputtered films. It was found that uncoated and titanium-oxide-coated glass require more silver to achieve the same low value of sheet resistance than silver on zinc-oxide-coated glass. This can be associated with the energy of interaction between surface and silver atoms. Silver films made by cathodic arc deposition show an earlier onset of island coalescence and formation of short links. It was found that silver islands in energetic deposition exhibit a reduced aspect ratio compared to evaporation and sputtering. A nominal $0.1 \mathrm{~nm}$ niobium underlayer increases the nucleation density and promotes coalescence of silver islands, however, a $0.2 \mathrm{~nm}$ layer did not show these features, indicating the need for further studies.
\end{abstract}




\section{Introduction}

Spectrally selective, transparent coatings are of great interest for energy-efficient window coatings. In cold climates, low-emittance (low-E) coatings with high solar transmittance $(0.3<\lambda<3$ $\mu \mathrm{m})$ and high thermal infrared (IR) reflectance $(3<\lambda<100 \mu \mathrm{m})$ are required, while in warm climates solar control coatings of high luminous transmittance $(0.4<\lambda<0.7 \mu \mathrm{m})$ and high near-infrared reflectance $(0.7<\lambda<3 \mu \mathrm{m})$ are beneficial. Both types of coatings can be obtained by embedding a thin metal film between anti-reflecting dielectric layers. ${ }^{1)}$ It is known that low-emittance and high infrared reflectance are directly related to the sheet resistance of the metal layer. ${ }^{2)}$ Silver is the metal of choice because of its high conductivity and color-neutral transmission in the visible range of the spectrum. To maximize transmittance in the visible and reflectance in the IR, it is desirable to produce a very thin silver film that is as conducting as possible.

It is known that silver grows on oxides in the Volmer-Weber mode, i.e. silver islands are formed before a continuous film is obtained. ${ }^{3)}$ Ultrathin silver films consisting of islands show relatively high absorption in the visible and low IR reflectance. Coalescence of silver islands is therefore crucial for obtaining the desired optical properties of the coating. Commercially, silver films of about $12 \mathrm{~nm}$ thickness are produced for low-E application on a very large scale by magnetron sputtering on oxide-coated flat glass. ${ }^{2)}$ There exists a number of studies on nucleation and growth of metals on oxides, e.g. ${ }^{1,4-7)}$. Whilst nucleation, island growth, and coalescence has been investigated for evaporated $^{8)}$ and sputtered ${ }^{9)}$ silver films, little is known about silver films synthesized by energetic condensation. In energetic condensation, ${ }^{10,11)}$ film-forming atoms or ions arrive at the substrate surface with hyperthermal energies, typically in the range of $10-100 \mathrm{eV}$, leading to subplantation and nanoscale thermal spikes around the arriving particle's impact location. ${ }^{12)}$ Filtered cathodic arc vacuum (FCVA) deposition is a prominent example for energetic condensation. Thin gold films deposited by FCVA deposition have a lower roughness than evaporated and sputtered films. ${ }^{13)}$ In this work we focus 
on the coalescence of silver on oxide surfaces produced by FCVA deposition. The effect of a very thin niobium underlayer on nucleation and growth of silver films was also investigated.

\section{Experimental}

The experimental setup is shown in Fig. 1. A miniature pulsed cathodic arc plasma source ${ }^{14)}$ with a cylindrical silver cathode of $6.25 \mathrm{~mm}$ diameter was operated with a 10 -stage pulse-forming network. ${ }^{15)}$ The arc current pulses had a rectangular shape with $1.2 \mathrm{kA}$ amplitude and $620 \mu$ pulse width, with a pulse repetition rate of $1.6 \mathrm{pps}$. The plasma source injected streaming silver plasma into a $90^{\circ}$ magnetic macroparticle filter. ${ }^{16)}$ This filter was an open solenoid used to remove microscopic debris ("macroparticles”) produced at cathode spots. Neutral silver vapor, if present in the flow, is also removed by the filter, and thus fully ionized silver plasma arrived at the substrates, which were located $200 \mathrm{~mm}$ from the filter exit. The silver plasma streaming velocity was $11,100 \mathrm{~m} / \mathrm{s}$, corresponding to an average kinetic energy of $70 \mathrm{eV}$, and the mean ion charge state was 2.1 , Ref. ${ }^{17)}$ The chamber was cryogenically pumped to a base pressure of about $10^{-4} \mathrm{~Pa}$; no process gas was needed or used.

Uncoated glass standard microscope slides, magnetron-sputtered zinc-oxide-coated glass, and titanium-oxide-coated glass were used as substrates. The samples were mounted on a water-cooled substrate holder. The substrate temperature was generally near room temperature although the surface

is subject to heating by the deposition process. It is known ${ }^{18)}$ that the $\mathrm{TiO}_{2}$ layer is amorphous while the ZnO layer is polycrystalline with $\{0001\}$ orientation.

Coalescence of silver islands can be detected by measuring the onset of electronic conduction. The film resistance was measured in-situ during deposition. For that purpose, two silver contact pads, approximately $1 \mu \mathrm{m}$ thick, were deposited on the $25 \mathrm{~mm}$ wide samples prior to the experiments. The contact pads were $25 \mathrm{~mm}$ apart and thus an area of $25 \mathrm{~mm}$ x $25 \mathrm{~mm}$ was defined. The resistance between the contact pads was measured using a Keithley 177 precision multimeter, directly giving the 
sheet resistance in Ohms per square. A possible effect of the measuring current was considered as discussed below.

The deposition rate was calibrated ex-situ by measuring step heights of a relatively thick film (100 nm) using a Dektak profilometer. The nominal thickness of ultrathin films can easily be determined by counting arc pulses under the assumption that the deposited film thickness is directly proportional to the number of arc pulses. One needs to stress that film thickness determined this way needs to be understood as nominal, especially at the beginning of the deposition process when the "film" actually consists of islands. The deposition rate was determined to be 0.023 nm/pulse, corresponding to about 1/10 of a monolayer per pulse, an instantaneous rate of $37 \mathrm{~nm} / \mathrm{s}$ and an average rate of $0.037 \mathrm{~nm} / \mathrm{s}$.

For comparison, ultrathin silver films have also been deposited in the same process chamber by DC-magnetron sputtering. The same kinds of substrates were placed $100 \mathrm{~mm}$ in front of a 3” sputter gun with a silver target. The argon pressure during sputtering was $66 \mathrm{mPa}$. The sputter power was 40 $\mathrm{W}$ with a target potential of $-390 \mathrm{~V}$, leading to a deposition rate of $0.75 \mathrm{~nm} / \mathrm{s}$.

To investigate the effect of underlayer on nucleation and growth of silver films, a niobium layer of either $0.1 \mathrm{~nm}$ or $0.2 \mathrm{~nm}$ nominal thickness was deposited prior to silver deposition using a pulsed cathodic arc plasma source with a Nb cathode. All the deposition conditions were same as the cathodic arc silver deposition but the number of arc pulses. The nominal thickness of the niobium layer was determined by calibration using a thicker layer as mentioned above. Since the Nb films were very thin it is conceivable, or even likely, that partial oxidation may have occurred before the silver layer was deposited, even when the film was kept in high vacuum environment.

The nucleation and growth of silver films deposited by FCVA and sputtering were examined by transmission electron microscopy (Jeol, JEM-200FXII) and atomic force microscopy (Digital 
Instruments, Nanoscope IIIa). For TEM observation, silver films were deposited on back-etched Si with $50 \mathrm{~nm}$ thick LPCVD amorphous $\mathrm{Si}_{3} \mathrm{~N}_{4}$ electron transparent membranes.

\section{Results}

The in-situ sheet resistance of the silver film decreased as expected with increasing amount of silver deposited. Fig. 2 shows the resistance as a function of the number of arc pulses for Ag deposited on plain glass, zinc-oxide-coated glass, and titanium-oxide-coated glass. The sheet resistance as a function of nominal thickness indicates the existence of three regions: (i) the region of individually dispersed clusters and islands, (ii) a transition region where short-link conduction starts, and (iii) a region where a continuous film is formed, gradually approaching bulk resistivity when the film thickness much exceeds the electron mean free path (Drude model). From Fig. 2 one can see that uncoated and titanium-oxide-coated glass require more silver to achieve the same low value of sheet resistance than the zinc oxide coated glass. This can be associated with the energy of interaction between surface and film atoms. If the interaction energy between the adatoms and the atoms on the surface of the substrate is lower than the energy between the adatoms themselves, the film will grow as

discrete islands centered on initial nucleation sites. ${ }^{19)}$ The interaction between the zinc-oxide surface and the silver atoms is stronger than the interaction of silver with titanium-oxide-coated glass and uncoated glass. For this reason zinc-oxide-coatings are often used as substrate for low-emittance optical coatings obtained by sputtering.

The energetics of the deposition process (kinetic effects) and the substrate material and temperature (thermodynamic effects) are known to affect the transition region and formation of a continuous film. This is illustrated by the difference between the curves for cathodic arc and magnetron deposited silver on glass and $\mathrm{ZnO}$ as shown in Fig. 3. Nucleation and growth in energetic deposition are quailitatively different from nucleation and growth in conventional evaporation and sputtering 
because film-forming ions arrive with high kinetic energy sufficient for subplantation. Cathodic arc silver ions have an average kinetic energy of $70 \mathrm{eV}$, as compared to the $1-3 \mathrm{eV}$ in sputter deposition. Silver films made by cathodic arc deposition show an earlier onset of island coalescence and formation of short links. This indicates that silver islands in energetic deposition exhibit a reduced aspect ratio when compared to evaporation and sputtering, which can be linked to the subplantation growth mechanism. To confirm this, morphologies of cathodic arc-deposited and sputter-deposited silver islands were observed by TEM. Fig. 4 shows TEM micrographs of the filtered arc-deposited and the sputtered silver film, with $10 \Omega / \square$ of sheet resistance, each, at 8 and $16 \mathrm{~nm}$ in thickness, respectively. It can be seen that small and fine islands were formed in the arc-deposited silver layer, as opposed to the sputtered film, which shows coarse islands of irregular size.

Fig. 5 shows the sheet resistance of filtered arc deposited silver films on titanium-oxide-coated glass and with $\mathrm{Nb}$ underlayer of different thickness. Sheet resistance curves of silver films on $\mathrm{Nb}$ underlayer show faster transition and onset of electronic conduction that silver films without a $\mathrm{Nb}$ underlayer. When the $\mathrm{Nb}$ thickness was $0.1 \mathrm{~nm}$, electronic conduction was measured to set on at about $7 \mathrm{~nm}$ of silver. However, when the nominal thickness of the $\mathrm{Nb}$ underlayer was increased to $0.2 \mathrm{~nm}$, less improvement of electronic conduction of silver could be found. Fig. 6 shows the morphologies of silver islands formed on titanium-oxide-coated glass with a $\mathrm{Nb}$ underlayer of different thickness. It can be seen that the introduction of a $0.1 \mathrm{~nm} \mathrm{Nb}$ underlayer promotes a high nucleation density and early coalescence of silver islands. This indicates that silver islands on a $0.1 \mathrm{~nm}$ Nb layer exhibit a reduced aspect ratio, as shown in Table 1. However, silver islands were larger and the films rougher in the case of nominal $0.2 \mathrm{~nm} \mathrm{Nb}$ underlayer.

\section{Conclusions}


Uncoated and titanium-oxide-coated glass require more silver to achieve the same low value of resistance than zinc-oxide-coated glass. This can be related to the interaction energy between surface and silver atoms. The differences in kinetic energy between filtered cathodic arc deposition and magnetron sputtering lead to distinctly different film properties. Silver films made by cathodic arc deposition show an earlier onset of island coalescence and formation of short links. Silver islands in energetic deposition exhibit a reduced aspect ratio compared to evaporation and sputtering. The interaction energy between surface atoms and film atoms are important, and it was shown that a

niobium layer of nominal only $0.1 \mathrm{~nm}$ thickness can affects nucleation and coalescence of silver. While a $0.1 \mathrm{~nm}$ film of $\mathrm{Nb}$ promotes coalescence of silver, slightly thicker $\mathrm{Nb}$ films do not confirm this trend. Further studies are needed to clarify this situation, and also the role of possible oxygen contamination of niobium needs to be taken into account.

\section{Acknowledgments}

This work was supported by the Assistant Secretary for Energy Efficiency and Renewable Energy, Office of Building Technology, U.S. Department of Energy under Contract No. DE-AC03-76SF00098. Eungsun Byon was supported by the post-doctoral fellowship program of the Korean Science and Engineering Foundation (KOSEF). 


\section{References}

1 G. B. Smith, G. A. Niklasson, J. S. E. M. Svensson, and C. G. Granqvist, J. Appl. Phys. 59, 571 (1986).

2 H. J. Gläser, Large Area Glass Coating (Von Ardenne Anlagentechnik GmbH, Dresden, Germany, 2000).

3 R. Lazzari and J. Jupille, Surface Sci. 482-485, 823 (2001).

4 S. Xu and B. L. Evans, J. Mat. Sci. 27, 3108 (1992).

5 A. I. Maaroof and B. L. Evans, J. Appl. Phys. 76, 1047 (1994).

6 M. Avrekh, O. R. Monteiro, and I. G. Brown, Appl. Surf. Sci. 158, 217 (2000).

7 E. V. Barnat, D. Nagakura, P.-I. Wang, and T.-M. Lu, J. Appl. Phys. 91, 1667 (2002).

8 B. Gergen, H. Nienhaus, W. H. Weinberg, and E. M. McFarland, J. Vac. Sci. Technol. B 18, 2401 (2000).

$9 \quad$ M. Arbab, Thin Solid Films 381, 15 (2001).

10 O. R. Monteiro, Annual Rev. Mat. Sci. 31, 111 (2001).

11 J. S. Colligon, J. Vac. Sci. Technol. A 13, 1649 (1995).

12 A. Anders, Appl. Phys. Lett. 80, 1100 (2002).

13 A. Bendavid, P. J. Martin, and L. Wieczorek, Thin Solid Films 354, 169 (1999).

14 R. A. MacGill, M. R. Dickinson, A. Anders, O. R. Monteiro, and I. G. Brown, Rev. Sci. Instrum. 69, 801 (1998).

15 A. Anders, R. A. MacGill, and T. A. McVeigh, Rev. Sci. Instrum. 70, 4532 (1999).

16 A. Anders, Surf. Coat. Technol. 120-121, 319 (1999).

17 A. Anders and G. Y. Yushkov, J. Appl. Phys. 91, 4824 (2002).

18

R. Dannenberg, D. Glenn, and E. Stach, Tech. Conf. of Society of Vacuum Coaters, Philadelphia, 2001, pp. 218-224.

19 W. Sainty, Protection of optical surface by thin films. PhD Thesis, University of Sydney, 1994. 
Table 1. Roughness of silver islands formed on titanium-oxide-coated glass and $\mathrm{Nb}$ underlayer with different thickness.

\begin{tabular}{|c|c|c|c|}
\hline & $\mathrm{R}_{\mathrm{a}}(\mathrm{nm})$ & $\mathrm{R}_{\mathrm{q}}(\mathrm{nm})$ & $\mathrm{R}_{\max }(\mathrm{nm})$ \\
\hline $\mathrm{Ag} / \mathrm{TiO}_{2}$ & 2.2 & 2.9 & 27.6 \\
\hline $\mathrm{Ag} / \mathrm{Nb}(0.1 \mathrm{~nm}) / \mathrm{TiO}_{2}$ & 0.65 & 0.81 & 5.85 \\
\hline $\mathrm{Ag} / \mathrm{Nb}(0.2 \mathrm{~nm}) / \mathrm{TiO}_{2}$ & 2.33 & 3.2 & 30.34 \\
\hline
\end{tabular}




\section{Figure Captions}

FIG. 1 Experimental setup for filtered cathodic vacuum (FCVA) arc deposition of thin metal films.

FIG. 2 Sheet resistance of filtered arc deposited silver films on different substrates, which is expressed as nominal film thickness, measured immediately after completion of deposition.

FIG. 3 Sheet resistance of sputtered and filtered arc deposited silver films as a function of incident silver dose, which is expressed as nominal film thickness, measured immediately after completion of deposition.

FIG. 4 TEM micrographs of a) filtered arc deposited and b) sputtered silver films with 10 ohm of sheet resistance at 8 and $16 \mathrm{~nm}$ thickness, respectively.

FIG. 5 Sheet resistance of filtered arc deposited silver films on $\mathrm{TiO}_{2}$ substrate and $\mathrm{Nb}$ underlayer with different thickness.

FIG. 6 AFM images of filtered arc deposited silver films on $\mathrm{TiO}_{2}$ substrate and $\mathrm{Nb}$ underlayer with different thickness; a) no underlayer, b) $0.1 \mathrm{~nm}$ of $\mathrm{Nb}$ underlayer and c) $0.2 \mathrm{~nm}$ of $\mathrm{Nb}$ underlayer. 


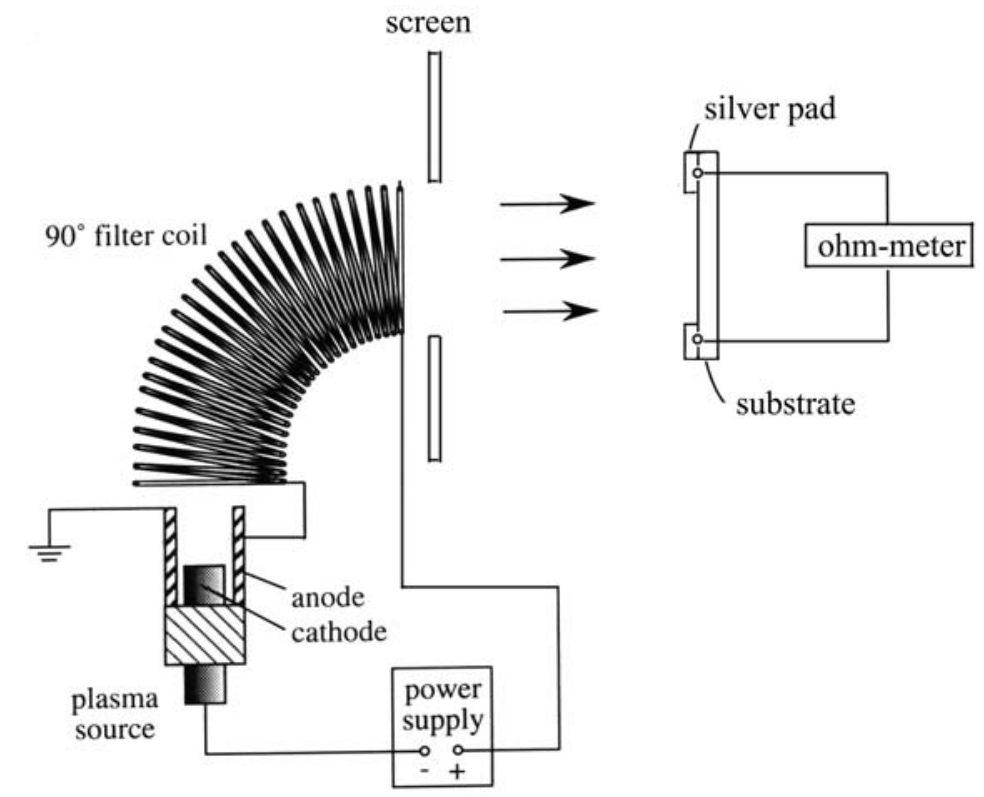

Fig. 1, B2-7

(Byon et. al.) 


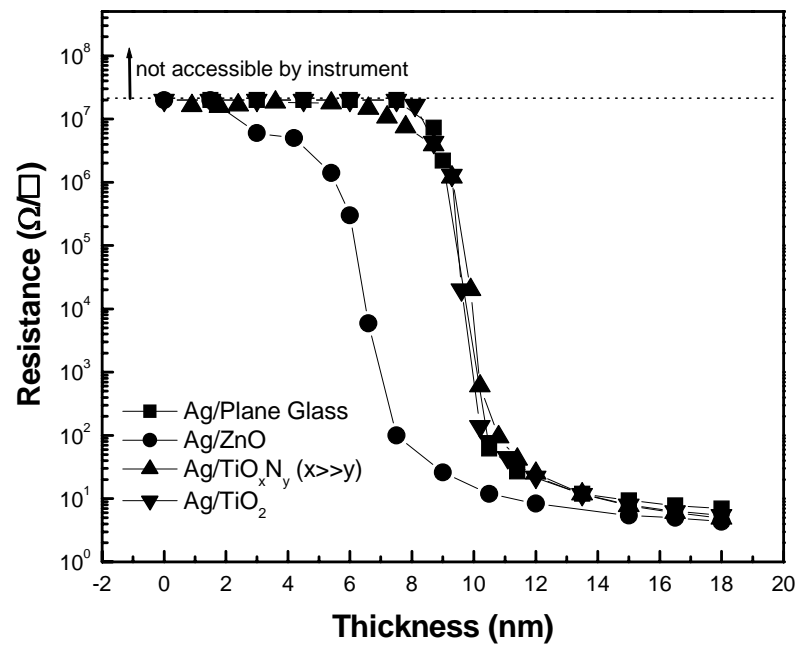

Fig. 2, B2-7

(Byon et. al.) 


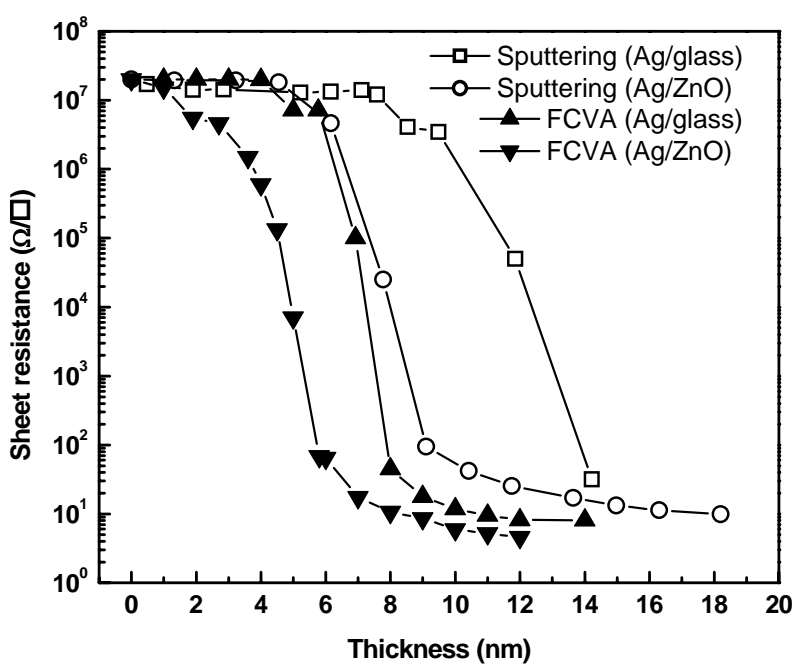

Fig. 3, B2-7

(Byon et. al.) 

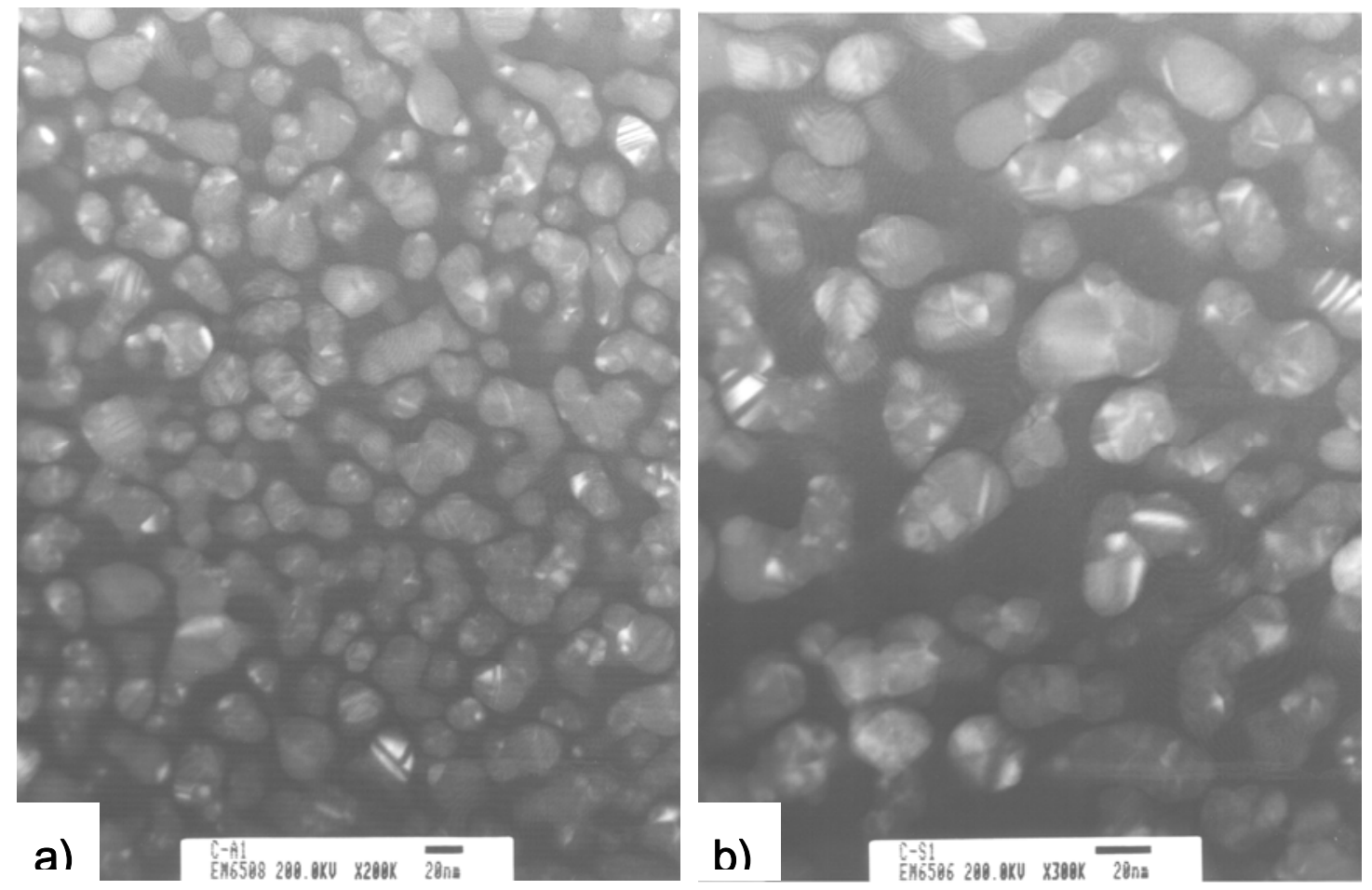

Fig. 4, B2-7

(Byon et. al.) 


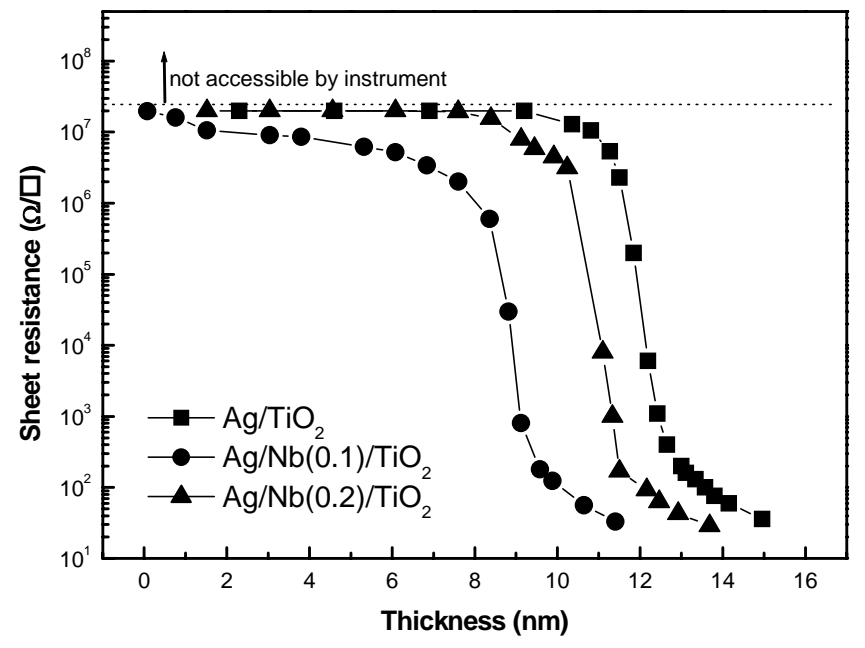

Fig. 5, B2-7

(Byon et. al.) 

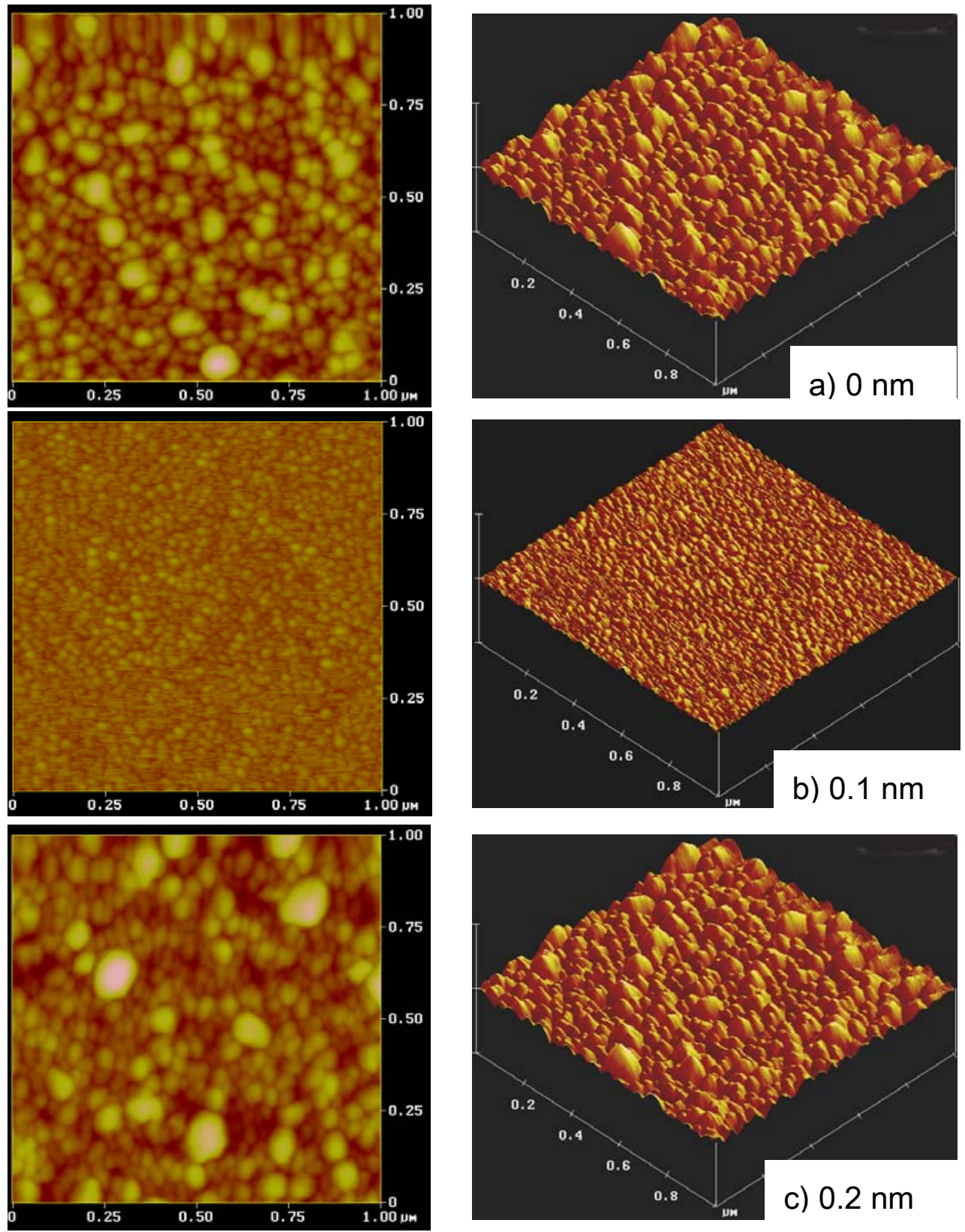

Fig. 6, B2-7

(Byon et. al.) 